\title{
Evaluation of a chemiluminescent enzyme-linked immunosorbent assay for the diagnosis of Trypanosoma cruzi infection in a nonendemic setting
}

\author{
Luis Izquierdo ${ }^{1 /+}$, Alexandre Ferreira Marques², Montserrat Gállego ${ }^{3}$, Sílvia Sanz ${ }^{1}$, Sílvia Tebar ${ }^{3}$, \\ Cristina Riera', Llorenç Quintó1, Edelweiss Aldasoro', Igor C Almeida², Joaquim Gascon'1
}

\author{
${ }^{1}$ Hospital Clinic, Barcelona Centre for International Health Research ${ }^{3}$ Laboratorio de Parasitología, Facultad de Farmacia, \\ Universitat de Barcelona, Barcelona, Spain ${ }^{2}$ Border Biomedical Research Center, University of Texas at El Paso, El Paso, Texas, USA
}

The disappearance of lytic, protective antibodies (Abs) from the serum of patients with Chagas disease is accepted as a reliable indicator of parasitological cure. The efficiency of a chemiluminescent enzyme-linked immunosorbent assay based on a purified, trypomastigote-derived glycosylphosphatidylinositol-anchored mucin antigen for the serologic detection of lytic Abs against Trypanosoma cruzi was evaluated in a nonendemic setting using a panel of 92 positive and 58 negative human sera. The technique proved to be highly sensitive $[100 \%$; 95\% confidence interval $(C I)=96-100]$ and specific (98.3\%; 95\% CI =90.7-99.7), with a kappa score of 0.99. Therefore, this assay can be used to detect active T. cruzi infection and to monitor trypanosomicidal treatment.

Key words: Chagas disease - lytic anti- $\alpha$-Gal antibodies - Trypanosoma cruzi

American trypanosomiasis, or Chagas disease, is a chronic infection caused by the protozoan parasite Trypanosoma cruzi. The disease can be transmitted by triatomine insect vectors, blood transfusion, organ transplantation, tainted foods and fluids and transplacentally. The acute phase of Chagas disease lasts six-eight weeks during which certain patients have fever, lymphadenopathy, splenomegaly and/or oedema, though most cases are asymptomatic or oligosymptomatic. In the event that the acute phase ends and the parasite is not completely eliminated, T. cruzi infection passes into a clinically silent chronic phase designated as the indeterminate clinical form. Although most infected individuals remain asymptomatic for life, $20-35 \%$ of patients develop severe chronic Chagas disease over a period of 10-30 years that is characterised by cardiac and/or gastrointestinal tract disorders. During the chronic phase of the disease, the levels of circulating parasite are far below the threshold of microscopic detection and the diagnosis is therefore primarily based on conventional serology.

Due to migration trends, there are millions of people from Chagas disease-endemic countries now living in North America, Europe, Australia and Japan, including thousands of people with T. cruzi infection. Congeni-

doi: 10.1590/0074-0276130112

Financial support: NIH (R01AI070655-A5, R01AI070655-A5S1, 5G12RR008124-16A1, 5G12RR008124-16A1S1, G12MD007592), AGAUR (2009SGR385) (to CRESIB)

LI and AFM contributed equally to this work.

+ Corresponding author: luis.izquierdo@cresib.cat

Received 22 June 2012

Accepted 19 June 2013 tal, transfusion-associated and/or transplant-associated transmission has been documented in the United States of America, Spain, Canada and Switzerland (Gascon et al. 2010). In the present paper, we describe the use of a chemiluminescent enzyme-linked immunosorbent assay (CL-ELISA) for the serodiagnosis of chagasic infection (Almeida et al. 1997) in our facilities at the Barcelona Centre for International Health Research. The test is based on the reactivity of serum with glycosylphosphatidylinositol (GPI)-anchored mucins purified from mammalian cell culture-derived trypomastigotes (tGPI-mucins) of the Y strain (Almeida et al. 1994). These antigens are specifically recognised by protective chagasic anti- $\alpha$-galactosyl antibodies (anti- $\alpha$-Gal Abs) and can be used to diagnose active disease because the Abs elicit both complement-dependent and complementindependent lysis of infective parasite stages, which revert after T. cruzi elimination (Almeida et al. 1991, 1993, 1997, Andrade et al. 1996, 2004, Pereira-Chioccola et al. 2000). Therefore, the assay combines the use of a highly specific antigen from the infective (trypomastigote) form of the parasite with a highly sensitive procedure for the detection of positive reactions and allows the detection of lytic anti- $\alpha$-Gal Abs in the serum of Chagas disease patients (Andrade et al. 1996, 2004, Almeida et al. 1997, de Marchi et al. 2011).

tGPI-mucins were purified by solvent extraction and hydrophobic interaction chromatography, as previously described (Almeida et al. 1994). CL-ELISA was performed as previously described (Almeida et al. 1997). Briefly, purified tGPI-mucins $(50 \mu \mathrm{L}$ at $0.32 \mathrm{pg} / \mu \mathrm{L})$ in $50 \mathrm{mM}$ carbonate-bicarbonate buffer $(\mathrm{pH} 9.5)$ were immobilised onto white, opaque 96-well CL-ELISA microplate wells (Nunc, Roskilde, Denmark). After an overnight incubation at $4^{\circ} \mathrm{C}$, free microplate binding sites were blocked with $5 \%$ bovine serum albumin (BSA) in phosphate-buffered saline (PBS), pH 7.4. The plates were 
incubated with $50 \mu \mathrm{L}$ human serum at a 1:800 dilution in PBS containing $1 \%$ BSA (PBS-BSA). The plates were then sequentially incubated with $50 \mu \mathrm{L}$ biotinylated anti-human immunoglobulin $\mathrm{G}$ (1:2,000 dilution) (Amersham, GE Healthcare Life Sciences, Buckinghamshire, UK) in PBS-BSA and $50 \mu \mathrm{L}$ streptavidin-horseradish peroxidase conjugate (1:1,000 dilution) (Amersham, GE Healthcare Life Sciences) in PBS-BSA. All the incubation steps were performed at $37^{\circ} \mathrm{C}$ for $1 \mathrm{~h}$. The plates were washed four times with PBS containing $0.05 \%$ Tween 20 after each incubation period. The reaction was developed with $50 \mu \mathrm{L}$ enhanced CL reagent (Pierce, Thermo Scientific) diluted 1:10 in $50 \mathrm{mM}$ carbonatebicarbonate buffer ( $\mathrm{pH}$ 9.5). The chemiluminescence measurements, expressed as relative luminescence units (RLUs), were performed in a multi-mode microplate reader (Synergy HT, Biotek). In each plate, we included a positive control (pool of 10 sera of confirmed chronic Chagas disease patients), a negative control (pool of 10 healthy donor sera from Chagas disease-endemic areas) and a background control (incubation buffer with no sera added), which were assayed in triplicate. Statistically valid cut-off values for the CL-ELISA results were calculated by defining the upper prediction limit, which was expressed as the standard deviation (SD) multiplied by a factor according to the number of negative controls and confidence level (99.5\%) and calculated based on the Student $t$ distribution (Frey et al. 1998). Thus, for each plate in which three negative controls were included (pool of 10 healthy donor sera), the cut-off values were established as the negative control mean plus 11.46 times the $\mathrm{SD}$ [cut-off value $=($ negative control mean $)+11.46$ $x(S D)]$ (Frey et al. 1998). The titre of each CL-ELISA is defined as the ratio of the tested serum's RLU value to the cut-off value. A serum sample was considered positive when its titre was equal to or higher than 1.0 and negative when the titre was equal or less than 0.9. Inconclusive or doubtful results showed titres above 0.9 or below 1.0 (Almeida et al. 1997). All the sera were tested in duplicate and the reported results are the means of two simultaneous determinations.

Negative serum samples from blood donors $(\mathrm{n}=58)$ were obtained from the Catalonian Blood Bank and were assayed for the presence of $T$. cruzi Abs using two commercially available Chagas Ab tests: ID-PaGIA (DiaMed, Cressier sur Morat, Switzerland) and Chagas Bioelisa Assay (Biokit, Lliçà d'Amunt, Spain) (Piron et al. 2008). Negative results were confirmed with an ELISA using crude antigen from $T$. cruzi epimastigotes (TC-Maracay strain) (Riera et al. 2009). Positive samples $(n=92)$ were obtained from the serum collection of the Laboratory of Parasitology, Faculty of Pharmacy, University of Barcelona. The samples were tested for T. cruzi using a specific polymerase chain reaction assay and two ELISAs: Chagas Bioelisa Assay and the technique using crude antigen from T. cruzi (Riera et al. 2009). Eighty-two of the positive patients $(89.1 \%)$ presented the indeterminate form of chronic Chagas disease and the other 10 subjects had cardiac and/or digestive manifestations. The serum samples were obtained before benznidazole treatment and, in all cases, both positive and negative donors signed an informed consent form approved by the Institutional Review Board of the Hospital Clinic of Barcelona.

The substantial number of inconclusive diagnoses of Chagas disease shows the need for a valid and widely accepted standard diagnostic procedure (Reithinger et al. 2009). As the available diagnostic procedures vary in sensitivity, the use of two diagnostic tests is generally recommended to confirm clinical suspicion. Because the samples used were previously confirmed as positive or negative by at least two different tests, the diagnostic performance characteristics of CL-ELISA using tGPI-mucins were calculated by employing those previous determinations as a reference. The measured end points were the number of true-positive (TP), true-negative (TN), false-positive (FP) and false-negative (FN) samples. Sensitivity was calculated as $\mathrm{TP} /(\mathrm{TP}+\mathrm{FN})$ and specificity was calculated as TN/(TN+FP). The positive predictive value (PPV), i.e., the proportion of patients with positive results who were correctly diagnosed, was calculated as TP/(TP+FP). The negative predictive value (NPV), which is the proportion of patients with negative results who were correctly diagnosed, was calculated as $\mathrm{TN} /(\mathrm{TN}+\mathrm{FN})$. The test efficiency, which is the proportion of all tests that gave correct results, was defined as
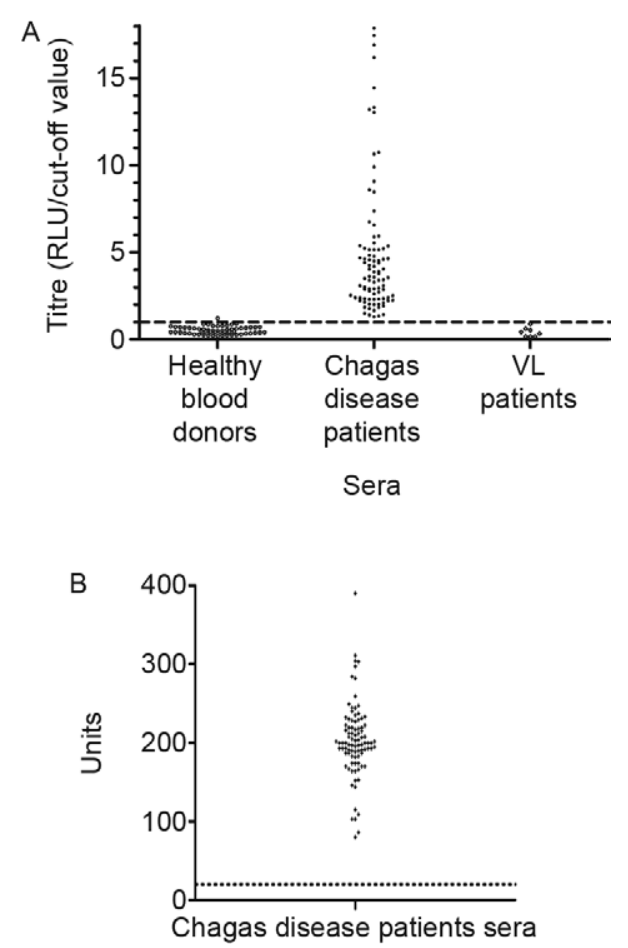

Reactivity of the chemiluminescent enzyme-linked immunosorbent assay (ELISA) using Trypanosoma cruzi glycosylphosphatidylinositol-mucins. The titres of sera of healthy blood donors $(n=58)$, chronic Chagas disease patients $(n=92)$ and patients with visceral leishmaniasis $(V L)(n=8)$ are indicated. Dotted line: cut-off value equivalent to titre $=1$ (A). Reactivity of the positive sera in an ELISA using crude antigen from $T$. cruzi epimastigotes. Dotted line: cut-off value established at 20 units (B) $(492 \mathrm{~nm}$ ratio between samples and calibrator pool). RLU: relative luminescence unit. Source: Riera et al. (2009). 
$(\mathrm{TP}+\mathrm{TN}) /($ number of all tests). Cohen's kappa coefficient, which reflects the concordance between different tests, was calculated as follows: (Ao-Ae)/(1-Ae), with Ao being the observed agreement and Ae the agreement expected by chance.

Fifty-seven of 58 negative serum samples were negative by CL-ELISA. Only one healthy blood donor serum sample showed a titre immediately above the cut-off line (titre $=1.2$ ) and was thus considered positive in the assay. Of the 92 positive sera analysed, 92 were positive by our CL-ELISA (A in Figure). Therefore, the sensitivity and specificity of this technique in detecting Chagas disease were $100 \%$ [95\% confidence interval $(\mathrm{CI})=96-100]$ and $98.3 \%(95 \% \mathrm{CI}=90.7-99.7)$, respectively. PPV and NPV were $98.9 \%$ and $100 \%$, respectively, and the percentage of sera correctly classified was $99.3 \%$. The kappa test score was $0.99(95 \% \mathrm{CI}=0.96-1.00)$. Thus, as previously reported (Almeida et al. 1997, de Marchi et al. 2011), CLELISA using tGPI-mucins has a high efficiency and discriminatory capacity. Importantly, the assay also shows a very high level of agreement with classic serology and other Chagas disease diagnostic tests. Only two (2.2\%) of the 92 positive samples correctly tested by CL-ELISA showed a titre below 1.5, which might be considered too close to the assay cut-off to produce negative results when repeated. However, in those two cases, the replicates were always positive and presented values above the cut-off.

Due to the well-known cross-reactivity of chagasic sera with other organisms, mainly the closely related members of the Trypanosomatidae family, eight serum samples from patients affected with visceral leishmaniasis (VL) were tested using CL-ELISA; the results were negative in all of these cases (Figure). Thus, as previously reported in several studies, tGPI-mucins do not cross-react with sera from patients with VL or cutaneous leishmaniasis (Almeida et al. 1993, 1997, de Marchi et al. 2011). Therefore, this test could definitely be used in geographic regions in which leishmaniasis is endemic, such as Spain.

As previously described, the tGPI-mucin CL-ELISA test shows very high sensitivity and specificity values (Almeida et al. 1997). These values are comparable to those achieved using $T$. cruzi crude lysates (Riera et al. 2009), as observed in Figure (B), for mixtures of certain specific parasite recombinant antigens (Umezawa et al. 2003, Hernández et al. 2010, Longhi et al. 2012) and for other sensitive and specific confirmatory tests for Chagas disease, such as the trypomastigote excreted-secreted antigen blotting test (Umezawa et al. 1996). The high stability of tGPI-mucin antigen gives us the opportunity to use this unconventional technique in a nonendemic setting without any significant decrease in reactivity. In addition, it has been reported elsewhere that, together with other criteria, a tGPI-mucin antigenic preparation may be used as a criterion for cure in treated patients (Almeida et al. 1993, Andrade et al. 1996, 2004), as the assay can replace the in vitro determination of parasite lysis. This concept adds value to the use of the technique in our facilities.

It is widely accepted that conventional serologic assays should be the basis for the evaluation of drug efficacy against $T$. cruzi because the only possible reason for a negative result would be the disappearance of the stimulus for specific $\mathrm{Ab}$ production: the parasite. However, after successful treatment, the results of conventional serologic assays remain positive for years or even decades during the chronic phase of Chagas disease, for reasons that are not yet well understood. These reasons most likely involve recurrent, nonspecific polyclonal activation and thus nonspecific, low-titre Abs (Almeida et al. 1993, 1997, Travassos \& Almeida 1993, Andrade et al. 1996, 2004, Rassi \& Luquetti 2003). The Ab-dependent lysis of infective $T$. cruzi stages by patient serum is a more reliable indicator of ongoing active infection and detects lytic Abs in which the immunodominant epitopes present on the surface of living trypomastigotes are recognised (Travassos \& Almeida 1993, Krettli 2009). Nevertheless, complement-mediated and complement-independent trypanolytic assays are not routine methods because these assays require the careful management of living infective trypomastigotes. Thus, the disappearance of specific lytic Abs has been advocated as a suitable indicator of parasitological cure (Krettli et al. 1982, Almeida et al. 1991, 1993, Galvão et al. 1993). A complete correlation $(100 \%)$ has been reported between chagasic serum lytic activity and the recognition of the purified trypomastigote surface glycoprotein 160 in an immunoassay (Krettli 2009).

The present report shows an evaluation of the sensitivity and specificity of CL-ELISA using tGPI-mucins for the serodiagnosis of Chagas disease in a panel of human sera at our facilities. This assay has been previously employed to evaluate the efficacy of a 60-day course of benznidazole treatment in children with early chronic $T$. cruzi infection (Andrade et al. 2004) and to diagnose active infection in samples from blood banks in South America (Almeida et al. 1997, de Marchi et al. 2011). We propose the use of CL-ELISA with purified tGPI-mucins or synthetic $\alpha$-Gal-containing glycans recognised by the lytic anti- $\alpha$-Gal Abs (Almeida et al. 1994) in further research studies to assess the effectiveness of Chagas disease chemotherapy in both endemic and nonendemic settings.

\section{ACKNOWLEDGEMENTS}

To Hernando A del Portillo, for support, reagents and helpful scientific discussions throughout this work, to Ruth Aguilar, for helpful technical discussions, and to the Biomolecule Analysis Core Facility at the Border Biomedical Research Center, UTEP.

\section{REFERENCES}

Almeida IC, Covas DT, Soussumi LM, Travassos LR 1997. A highly sensitive and specific chemiluminescent enzyme-linked immunosorbent assay for diagnosis of active Trypanosoma cruzi infection. Transfusion 37: 850-857.

Almeida IC, Ferguson MA, Schenkman S, Travassos LR 1994. Lytic anti-alpha-galactosyl antibodies from patients with chronic Chagas disease recognize novel O-linked oligosaccharides on mucinlike glycosyl-phosphatidylinositol-anchored glycoproteins of Trypanosoma cruzi. Biochem J 304: 793-802.

Almeida IC, Krautz GM, Krettli AU, Travassos LR 1993. Glycoconjugates of Trypanosoma cruzi: a $74 \mathrm{kD}$ antigen of trypomastigotes specifically reacts with lytic anti-alpha-galactosyl antibodies from patients with chronic Chagas disease. J Clin Lab Anal 7: 307-316. 
Almeida IC, Milani SR, Gorin PA, Travassos LR 1991. Complementmediated lysis of Trypanosoma cruzi trypomastigotes by human anti-alpha-galactosyl antibodies. J Immunol 146: 2394-2400.

Andrade ALSS, Martelli CM, Oliveira RM, Silva SA, Aires AI, Soussumi LM, Covas DT, Silva LS, Andrade JG, Travassos LR, Almeida IC 2004. Short report: benznidazole efficacy among Trypanosoma cruzi-infected adolescents after a six-year followup. Am J Trop Med Hyg 71: 594-597.

Andrade ALSS, Zicker F, de Oliveira RM, Silva SA, Luquetti A, Travassos LR, Almeida IC, de Andrade SS, de Andrade JG, Martelli CM 1996. Randomised trial of efficacy of benznidazole in treatment of early Trypanosoma cruzi infection. Lancet 348: 1407-1413.

de Marchi CR, Di Noia JM, Frasch AC, Amato Neto V, Almeida IC, Buscaglia CA 2011. Evaluation of a recombinant Trypanosoma cruzi mucin-like antigen for serodiagnosis of Chagas disease. Clin Vaccine Immunol 18: 1850-1855.

Frey A, Di Canzio J, Zurakowski D 1998. A statistically defined endpoint titer determination method for immunoassays. J Immunol Methods 221: 35-41.

Galvão LM, Nunes RM, Cançado JR, Brener Z, Krettli AU 1993. Lytic antibody titre as a means of assessing cure after treatment of Chagas disease: a 10 years follow-up study. Trans $R$ Soc Trop Med Hyg 87: 220-223.

Gascon J, Bern C, Pinazo MJ 2010. Chagas disease in Spain, the United States and other non-endemic countries. Acta Trop 115: 22-27.

Hernández P, Heimann M, Riera C, Solano M, Santalla J, Luquetti AO, Beck E 2010. Highly effective serodiagnosis for Chagas disease. Clin Vaccine Immunol 17: 1598-1604.

Krettli AU 2009. The utility of anti-trypomastigote lytic antibodies for determining cure of Trypanosoma cruzi infections in treated patients: an overview and perspectives. Mem Inst Oswaldo Cruz 104 (Suppl. I): 142-151.

Krettli AU, Cancado JR, Brener Z 1982. Effect of specific chemotherapy on the levels of lytic antibodies in Chagas disease. Trans R Soc Trop Med Hyg 76: 334-340.
Longhi SA, Brandariz SB, Lafon SO, Niborski LL, Luquetti AO, Schijman AG, Levin MJ, Gómez KA 2012. Evaluation of inhouse ELISA using Trypanosoma cruzi lysate and recombinant antigens for diagnosis of Chagas disease and discrimination of its clinical forms. Clin Vaccine Immunol 17: 1598-1604.

Pereira-Chioccola VL, Acosta-Serrano A, de Almeida IC, Ferguson MA, Souto-Padron T, Rodrigues MM, Travassos LR, Schenkman S 2000. Mucin-like molecules form a negatively charged coat that protects Trypanosoma cruzi trypomastigotes from killing by human anti-alpha-galactosyl antibodies. J Cell Sci 113: 1299-1307.

Piron M, Vergés M, Muñoz J, Casamitjana N, Sanz S, Maymó RM, Hernández JM, Puig L, Portús M, Gascon J, Sauleda S 2008. Seroprevalence of Trypanosoma cruzi infection in at-risk blood donors in Catalonia (Spain). Transfusion 48: 1862-1868.

Rassi A, Luquetti AO 2003. Specific treatment for Trypanosoma cruzi infection (Chagas disease). In KM Tyler, MA Miles (eds.), American trypanosomiasis, Springer, New York, p. 117-125.

Reithinger R, Tarleton RL, Urbina JA, Kitron U, Gurtler RE 2009. Eliminating Chagas disease: challenges and a roadmap. BMJ 338: b1283.

Riera C, Vergés M, López-Chejade P, Piron M, Gascon J, Fisa R, Gállego M, Portús M 2009. Desarrollo y evaluación de una técnica ELISA con antígeno crudo de Trypanosoma cruzi para el diagnóstico de la enfermedad de Chagas. Enferm Emerg 11: 22-29.

Travassos LR, Almeida IC 1993. Carbohydrate immunity in American trypanosomiasis. Springer Semin Immunopathol 15: 183-204.

Umezawa ES, Bastos SF, Coura JR, Levin MJ, Gonzalez A, Rangel-Aldao R, Zingales B, Luquetti AO, da Silveira JF 2003. An improved serodiagnostic test for Chagas disease employing a mixture of Trypanosoma cruzi recombinant antigens. Transfusion 43: 91-97.

Umezawa ES, Nascimento MS, Kesper Jr N, Coura JR, Borges-Pereira J, Junqueira AC, Camargo ME 1996. Immunoblot assay using excreted-secreted antigens of Trypanosoma cruzi in serodiagnosis of congenital, acute and chronic Chagas disease. J Clin Microbiol 34: 2143-2147. 